\title{
Fluidic vision
}

\section{Liquid waveguides, deformable lenses, microdroplet lasers and biosensors are all technologies based on optofluidics. Now the field may even be able to help with issues such as energy production.}

This month, Nature Photonics presents a Focus Issue dedicated to the latest advances in optofluidics. Although the term 'optofluidics' is less than 10 years old, the combination of light and fluids has proved to be of great benefit. Researchers are now finding applications of optofluidics that range from imaging, particle control and the detection of chemical and biological agents, through to enhancing photonic circuits and energy generation.

According to Yeshaiahu Fainman at the University of California San Diego, who recently published a book on the topic ${ }^{1}$, optofluidics combines optics and photonics with microfluidics across a wide range of size scales to advance numerous areas of science and engineering. He told Nature Photonics that the term 'optofluidics' most likely first appeared in the name of a research centre funded by the Defense Advanced Research Projects Agency in 2003, but has evolved significantly since then. The centre was established to "develop adaptive optical circuits and systems by integrating optical and fluidic devices." Optofluidics soon found plasmonic, biomedical sensing and energy applications. At an early stage, it became evident that microfluidics could bring a wide range of advantages to the field of optofluidics, including the ability to change the optical properties of an optofluidic device by changing the fluid contained within, developing optically smooth interfaces between fluids to reduce scattering losses, and the ability to create gradients in optical properties using streams of miscible fluids.

"A review ${ }^{2}$ by Demetri Psaltis, Stephen Quake and Changhuei Yang led to a more symmetric definition of optofluidics, in which the advantages of various optofluidic technologies were discussed as beneficial to both optics and microfluidics," explained Fainman. "The most appropriate description of optofluidics would be to broadly define it as the combination of optics and microfluidics in the same platform to leverage the specific advantages of these two disciplines."

Optofluidics has now evolved to include biosensing platforms, in which microfluidics is used to deliver

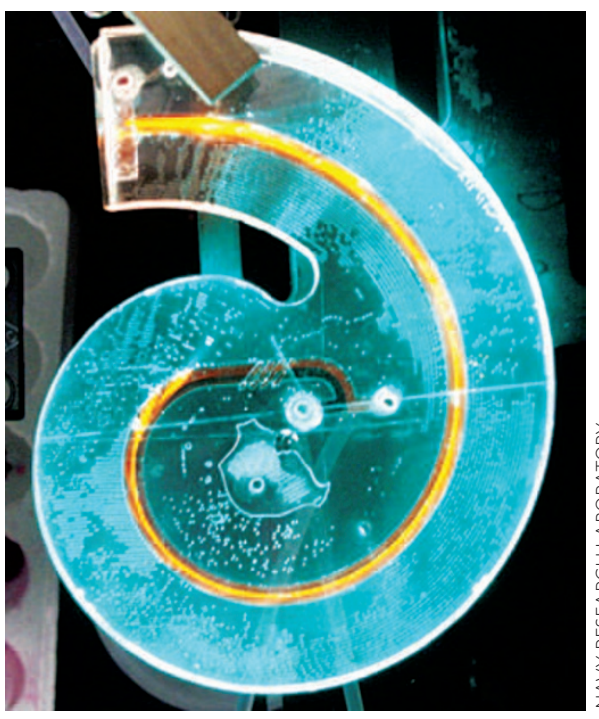

shaping, routing and switching, as well as optical manipulation and the analysis of particles in liquids. A Review by Xudong Fan and Ian White on page 591 summarizes research in the field of optofluidic sensing. Devices for refractive-index-based detection, fluorescence-based sensors and optofluidic surface-enhanced Raman spectroscopy are also covered.

One of the most recent and intriguing applications of optofluidics is for tackling the issues of energy production and storage. In their Review on page 583, David Erickson, David Sinton and Demetri Psaltis discuss optofluidics for energy applications, including energy transformation and fuel production in photobioreactors, photocatalytic and solar thermochemical reactions, and solar energy collection and control.

In an interview with Frances Ligler

biomolecules to novel functionalized optical and nanophotonic sensing devices and structures. This allows for the compact and dense integration of optofluidic technology in biomedical sensing, where it can be used to monitor multiple biochemical reactions in real-time.

"A major challenge for optofluidics is achieving a large interaction cross-section between the nanoparticles/biomolecules and the optical field. The current trend is to create nanophotonic metal-dielectric resonators that can localize optical modes to ultrasmall volumes, thereby providing a large volume overlap with the nanoparticle and thus providing large interaction cross-sections," explained Fainman. "The challenge is still how to bring the nanoparticles into close proximity with these localized modes. Recent efforts to create nanofluidic composite membranes have demonstrated great potential for co-localizing nanoparticles with these nanoscale resonators."

Optofluidics has also enabled the realization of an entirely new class of photonics elements. A Review by Holger Schmidt and Aaron Hawkins on page 598 highlights recent progress in the development of optofluidic-based photonic components. Such devices, based on the combination of fluidic media with integrated optical structures, have provided new techniques for light generation, from the Navy Research Laboratory on page 569 , we learn that researchers have been combining fluids and light long before the term 'optofluidics' was used. Ligler also expressed particular interest in the use of optofluidics for energy applications. "One question is whether optofluidic systems can be used on-chip to provide energy for very small systems, or even in vivo medical devices," said Ligler. She also discussed some of the practical issues associated with such systems, including material limitations.

Aside from the field's exciting potential for energy applications, it seems that the use of optofluidics to eliminate the need for optical connections, whether for remote sensing or for solving the major problem of connectivity in integrated devices, is a key research direction. Ligler pointed out that micro- and nanomanufacturing based on optofluidics, although still in its infancy, is already giving promising results. We are excited about the range and variety of potential optofluidic applications, but also look forward to future advances closer to home, in traditional photonics devices and components such as waveguides, resonators and optical circuits.

\footnotetext{
References

1. Fainman, Y., Psaltis, D., Lee, L. \& Yang, C. Optofluidics: Fundamentals, Devices and Applications (McGraw-Hill, 2009). 2. Psaltis, D., Quake, S. R. \& Yang, C. Nature 442, 381-386 (2006).
} 